Original Research

\title{
Transcatheter Intracerebral Laser Photobiomodulation Therapy for Treatment of the Consequences of Ischemic Stroke with Distal Atherosclerotic Lesion
}

Ivan V. Maksimovich *

Clinic of Cardiovascular Diseases named after Most Holy John Tobolsky, Moscow, Russia; E-Mail: carvasc@yandex.ru

* Correspondence: Ivan V. Maksimovich; E-Mail: carvasc@yandex.ru

Academic Editor: Jih-Huah Wu

Special Issue: Photobiomodulation Therapy

OBM Integrative and Complementary Medicine Received: July 24, 2021

2021, volume 6 , issue 4

Accepted: October 03, 2021

doi:10.21926/obm.icm.2104036

Published: October 21, 2021

\begin{abstract}
The present research is dedicated to the application of intracerebral laser PBMT for the treatment of the consequences of ischemic stroke. The effectiveness of intracerebral laser PBMT is compared to that of the conservative therapy methods. Recent studies have demonstrated a high potential of laser technology PBMT in the treatment of various ischemic lesions of the brain. A total of 836 patients who had experienced ischemic strokes of different degrees of severity in the period ranging from 6 months to 6 years prior to the treatment were included in the research. The age of these patients ranged between 29 and 81 years (average age: 74.9 years). There were 593 men (70.93\%) and 243 women (29.07\%). The patients were divided into two groups - the Test Group and the Control Group. The Test Group comprised $511(61.12 \%)$ patients with distal lesions of the intracerebral arteries. These patients underwent transcatheter intracerebral laser PBMT. The Control Group comprised 325 (38.88\%) patients with similar distal lesions of the intracerebral arteries. These patients, however, received the conservative treatment. The Test Group patients exhibited good clinical results in 259 (87.21\%) cases after small focal strokes, in 94 (60.26\%) cases after medium focal strokes, and in $12(20.69 \%)$ cases after macro-focal strokes. The Test Group
\end{abstract}

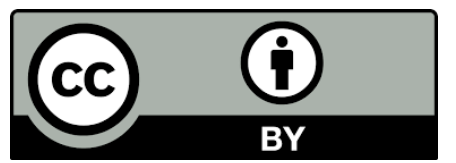

(c) 2021 by the author. This is an open access article distributed under the conditions of the Creative Commons by Attribution License, which permits unrestricted use, distribution, and reproduction in any medium or format, provided the original work is correctly cited. 
patients exhibited satisfactory clinical results in $33(11.11 \%)$ cases after small focal strokes, in 39 (25.00\%) cases after medium focal strokes, and- in 22 (37.93\%) cases after macro-focal strokes. In the Control Group, 51 (21.07\%) patients exhibited good clinical results after small focal strokes, while none of the patients exhibited good results after medium focal strokes and macro-focal strokes. In the Control Group, satisfactory clinical results were exhibited by $60(24.79 \%)$ patients after small focal strokes and $8(19.05 \%)$ patients after medium focal strokes, while none of the patients exhibited satisfactory results after macro-focal strokes. Transcatheter intracerebral laser PBMT is an effective, pathogenetically substantiated method for the treatment of the consequences of ischemic stroke. This method enables the restoration of daily-life activities and cognitive and mental functions, thereby enabling the patients to be fully active in their lives again.

\section{Keywords}

Cerebral ischemic stroke; transcatheter intracerebral laser PBMT; angiogenesis; neurogenesis; regeneration

\section{Introduction}

Ischemic stroke is one of the most common causes of severe disability and death in many countries across the world [1-3]. Just the US registers approximately 800 thousand cases of ischemic strokes each year $[2,3]$. In the acute cases, up to $30 \%$ of patients die immediately, while an additional $12 \%-15 \%$ of patients die in the 12 months following the stroke.

The brain requires the most blood among all organs in the human body and has, therefore, a complex system of angioarchitectonics and microcirculation [4, 5]. Large arteries deliver blood to the peripheral bed of the brain, while the intracerebral capillary bed plays a key role in the blood supply, oxygenation, and metabolism of neurons [4-8]. The cerebral tissue contains up to 3-4 thousand capillaries per cubic centimeter [9].

The tissues in the brain are extremely sensitive to circulatory disorders and progressing hypoxia, which develop rapidly into ischemia and neurodegenerative processes $[5,9,10]$. The larger the zone of ischemia and the greater it spreads to the different regions of the brain, the harder is the stroke experienced by the patient.

One of the main causes underlying an ischemic stroke is atherosclerosis, which is a systemic disease that affects both extracranial and intracerebral arteries, arterioles, and capillaries $[4,5,8$, 11-13]. Approximately $9.33 \%$ of the cases of atherosclerotic lesions are of the extracranial type, $46.19 \%$ of the cases are of the mixed type, and $44.53 \%$ of the cases are of the intracranial type [4].

As the ischemic processes progress, in addition to the arterial and capillary beds playing important roles in the blood supply, the collateral bed plays the role of allowing the blood to flow to the ischemic regions of the brain from the other unaffected vascular regions [4, 9]. The development of the collateral blood supply in the human brain is directly dependent on the level of the angiogenesis activity, which, in turn, stimulates the neurogenesis and regeneration processes in the cerebral tissue [5, 14-17]. The collateral blood supply usually develops as age progresses. Therefore, older patients with naturally developed collateral blood supply exhibit rapid progression 
of ischemic strokes compared to the younger patients with yet-to-be-developed developed collateral blood supply $[1,4,18]$.

Conservative methods of treating ischemic stroke mainly aim for vasodilation, improvement in microcirculation, and restoration of the metabolic processes occurring in the cerebral tissue [4, 5]. However, the effectiveness of the drugs used in such methods is currently limited [11, 18]. Therefore, conventional treatment methods are usually effective for small focal strokes with insignificant foci of neurodegeneration [5, 18], and for the extensive ischemic lesions, these treatment methods prove to be inadequate $[4,7,18]$.

Treatment with reconstructive and interventional surgeries is aimed at improving the main cerebral blood supply, and these methods are usually performed on large arterial branches. However, while these surgical methods have worked suitably on brachiocephalic arteries [19-25], these are difficult to perform in the case of distal intracranial atherosclerotic lesions due to the small diameter and the intracerebral location of the vessels [26, 27].

The methods of transcatheter intra-arterial thrombolysis and mechanical thrombectomy are also aimed at restoring the main cerebral blood supply, although it is advisable to perform these only in the acute cases of ischemic stroke [28-30].

The current scenario of treatment of the consequences of ischemic stroke warrants the development of novel, comprehensive, and effective methods aimed at improving the various pathological processes occurring during the development of the condition.

A highly promising approach in this regard is the application of laser energy in the treatment of ischemic stroke $[17,18]$.

One of the methods that involve laser energy application is photobiomodulation therapy (PBMT), which utilizes laser with low output power and red or near-infrared wavelengths (NIR) (spectral range $600-1100 \mathrm{~nm}$ ) [17, 31, 32-39]. PBMT involves the transcranial, intranasal, and transcatheter intracerebral methods of exposure.

Transcranial PBMT involves the practical non-invasive external introduction of laser energy into the brain through the bones of the skull [17, 31, 32].

In the intranasal PBMT, laser energy is introduced into the brain through the nasal cavities using non-invasive or slightly invasive techniques $[33,40]$. These two methods are often used together [33]. The third method of transcatheter intracerebral PBMT involves introducing the laser energy directly into the brain via the vascular system with minimum invasiveness, thereby preventing the loss of laser energy that occurs when it passes through the skin and the bones of the skull $[4,5,14$, $18,40,43]$.

In various experimental studies and clinical applications, these three methods have demonstrated high efficiency in the treatment of various cerebral disorders and the consequences of ischemic stroke [5, 31, 32, 36-44].

The present research is focused on comparing the effectiveness of the intracerebral transcatheter laser photobiomodulation therapy (PBMT) with the conservative methods in the treatment of the consequences of ischemic stroke with distal atherosclerotic lesions of the intracerebral arteries. 


\section{Materials and Methods}

\subsection{Ethical Statement}

All examinations, conservative treatment methods, and intracerebral transcatheter laser interventions conducted in the present research were approved by the Ethical Review Board (ERB) (Protocol No. 2 of 01-04-1996, Protocol No. 4 of 01-12-2002, and Protocol No. 12 of 03-26-2015). Consent of the included patients and their relatives was received in writing.

\subsection{Patient Selection Criteria for Test and Control Groups}

- Consent of patients and their relatives for conducting the necessary examinations and treatments;

- Somatic condition of the patients allowing to conduct the necessary examinations and treatments;

- Patient's medical history of having a cerebral ischemic stroke.

The present research examined the treatment of only those patients who had experienced an ischemic stroke with distal intracerebral atherosclerotic lesions in the period between 6 months and six years prior to receiving treatment.

Contraindications for the treatment in the patients of both test and control groups were pathologies such as severe disorders of the blood coagulation system and concomitant, infectious, or chronic diseases in acute stages.

\subsection{Patient Examination Plan for Test and Control Groups}

The patients underwent their first examination upon admission to the hospital and the subsequent one upon discharge [except for computed tomography (CT) and magnetic resonance imaging (MRI)]. Later, the patients were examined after the treatment, in the first year, at 6 months (including $\mathrm{CT}$ and $\mathrm{MRI}$ ) and then at 12-24 months (including CT and MRI).

The examination included:

- assessment of any changes in the cerebral structure using CT and MRI scans [the examination was conducted in different independent laboratories];

- assessment of daily-life activities based on functional evaluation using the Bartel Index (BI) [45];

- assessment of the clinical severity of dementia using the Clinical Dementia Rating scale (CDR) [46];

- the Mini-Mental State Examination (MMSE) to assess the cognitive functions [47];

- various laboratory tests conducted in accordance with the criteria of interventional neuroangiology;

- assessment of the cerebral blood flow and microcirculation using scintigraphy (SG);

- assessment of cerebral perfusion blood supply using rheoencephalography (REG);

- assessment of the intracerebral vascular and microcirculatory channel using cerebral multigated angiography (MUGA). The primary examination was conducted upon admission to the hospital, and the examination was repeated immediately after PBMT (for the test group 
patients) and then after an interval of 2-10 years after the therapy. In certain cases, MSCT angiography (MSCTA) or MR angiography (MRA) was also conducted.

A total of 836 patients with distal atherosclerotic lesions of the intracerebral arteries, whose medical history included ischemic strokes of a varying degree of severity, were selected. The age of the selected patients varied between 29 and 81 years (average age: 74.9 years). There were 593 men (70.93\%) and 243 women (29.07\%).

\subsubsection{Test Group}

- 511 (61.12\%) patients received the transcatheter intracerebral laser PBMT.

- 297 (58.12\%) patients had experienced small focal strokes;

- 156 (30.53\%) patients had experienced medium focal strokes;

- $58(11.35 \%)$ patients had experienced macro focal strokes.

\subsubsection{Control Group}

- 325 (38.88\%) patients received conservative treatment.

- $242(74.46 \%)$ patients had experienced small focal strokes;

- 42 (12.92\%) patients had experienced medium focal strokes;

- $41(12.62 \%)$ patients had experienced macro focal strokes.

All patients in both test and control groups had a similar somatic state, severity, and size of the post-ischemic focus. The timing of the stroke was also the same for all patients.

The results of the initial examination of the patients in the test group and the control group are presented in Table 1.

Table 1 Patient Examination Results.

\begin{tabular}{|c|c|c|}
\hline CHARACTERISTIC OF IDENTIFIED CHANGES & $\begin{array}{l}\text { Test Group } \\
\text { N-511 }\end{array}$ & $\begin{array}{l}\text { Control Group } \\
\mathrm{N}-325\end{array}$ \\
\hline \multicolumn{3}{|l|}{ Cerebral CT and MRI } \\
\hline $\begin{array}{l}\text { General involutive brain changes with enlargement of the } \\
\text { subarachnoid space }\end{array}$ & $429(83.95 \%)$ & $273(84.00 \%)$ \\
\hline Enlargement of the Sylvian fissures & $431(84.34 \%)$ & $275(84.62 \%)$ \\
\hline Signs of non-occlusive hydrocephalus & $317(62.04 \%)$ & $204(62.77 \%)$ \\
\hline Small focal strokes & $296(57.93 \%)$ & $242(74.46 \%)$ \\
\hline Medium focal strokes & $156(30.53 \%)$ & $42(12.92 \%)$ \\
\hline Macro focal strokes & $59(11.55 \%)$ & $41(12.62 \%)$ \\
\hline \multicolumn{3}{|l|}{ Daily Activity } \\
\hline Decrease in Barthel Index (BI) below 95 points & $280(54.79 \%)$ & $170(52.31 \%)$ \\
\hline Decrease in Barthel Index (BI) below 80 points & $135(26.41 \%)$ & $78(24.00 \%)$ \\
\hline Decrease in Barthel Index (BI) below 60 points & $53(10.37 \%)$ & $45(13.85 \%)$ \\
\hline \multicolumn{3}{|l|}{ Cognitive Disorders } \\
\hline Decrease to $20-25$ MMSE points & $332(64.97 \%)$ & $211(64.92 \%)$ \\
\hline Decrease to 12-19 MMSE points & $149(29.16 \%)$ & $95(29.23 \%)$ \\
\hline Decrease to 7-11 MMSE points & $30(5.87 \%)$ & $19(5.85 \%)$ \\
\hline
\end{tabular}




\begin{tabular}{|c|c|c|}
\hline \multicolumn{3}{|c|}{ Clinical Dementia Determination } \\
\hline CDR-1 & $312(61.06 \%)$ & $183(56.31 \%)$ \\
\hline CDR-2 & $141(27.59 \%)$ & $85(26.15 \%)$ \\
\hline CDR-3 & $31(6.07 \%)$ & $37(11.38 \%)$ \\
\hline \multicolumn{3}{|l|}{ Cerebral SG } \\
\hline Decreased blood flow in cerebral hemispheres & $511(100 \%)$ & $325(100 \%)$ \\
\hline \multicolumn{3}{|l|}{ REG } \\
\hline $\begin{array}{l}\text { Decreased volumetric pulse blood supply in the carotid } \\
\text { system }\end{array}$ & $511(100 \%)$ & $325(100 \%)$ \\
\hline \multicolumn{3}{|l|}{ Cerebral MUGA } \\
\hline $\begin{array}{l}\text { Stenotic lesions in the distal pools of intracerebral } \\
\text { branches }\end{array}$ & $408(79.84 \%)$ & $260(80.00 \%)$ \\
\hline $\begin{array}{l}\text { Occlusive lesions in the distal pools of intracerebral } \\
\text { branches }\end{array}$ & $511(100 \%)$ & $325(100 \%)$ \\
\hline Depletion of capillary contrast (capillary bed damage) & $511(100 \%)$ & $325(100 \%)$ \\
\hline Stenotic lesions in the main pools of intracerebral branches & - & - \\
\hline $\begin{array}{l}\text { Occlusive lesions in the main pools of intracerebral } \\
\text { branches }\end{array}$ & - & - \\
\hline
\end{tabular}

\subsection{Treatment Methods}

\subsubsection{Test Group}

In the catheterization laboratories (cath labs), cerebral multi-gated angiography (MUGA) was conducted under the effect of local anesthesia, in accordance with the Seldinger technique, with transfemoral access in frontal and lateral projections. Using the digital program named "Angio Vision", the intensity of the cerebral capillary blood supply was determined automatically $[4,5,41$ 44].

The guiding catheters are introduced into the common, internal carotid arteries and intracerebral branches under fluoroscopic control [4]. A flexible laser fiber-optic instrument is passed coaxially through these catheters for PBMT. This instrument has a diameter of 25-100 micrometers and a perpendicularly polished distal end that is connected to the laser system [4144]. The fiber-optic instrument is then made to reach the distal regions of the intracerebral arteries where laser irradiation has to be performed [41-44, 48].

PBMT was conducted manually using the ULF-01 continuous helium-neon laser ("Anod" manufacturing company, the city of Bryansk, Russia) $[43,48]$. The following were the parameters of the intracerebral transcatheter laser exposure: wavelength - 632.8 nanometers; laser output power - 25-45 mW; fiber output power - 24-44 mW; treatment session duration - 1200-2400 s; ray spot diameter of the vessel - 1-2 mm; and average dose during the treatment - 29-106 J. The depth of laser energy penetration into the cerebral tissues achieved using the transcatheter intracerebral method was $20-40 \mathrm{~mm}$. The interventions were performed on the affected side, as well as, prophylactically, on the contralateral side.

In order to achieve dynamic fluoroscopic control during the transcatheter interventions, a radiopaque substance (Omnipack 350) was administered, in small doses, periodically. After the 
endovascular treatment, repeated cerebral MUGA was performed using the Angio Vision digital program. This program automatically records the changes occurring in the vascular image in the corresponding phase of contrast in real-time $[4,5,42]$. The results reflect the state of the main cerebral blood supply and also the intensity of intracerebral angiogenesis and collateral and capillary revascularization $[5,43,44,48]$. If the blood supply is not restored completely after PBMT, the manipulation may be repeated. However, it was not required during the transcatheter interventions described in the present report.

After the completion of the intracerebral transcatheter laser PBMT, the patients received disaggregated, anticoagulant, vasodilating, antioxidant, and nootropic therapy, which is commonly accepted in interventional neuroangiology, including Aspirin, Heparin, non-direct anticoagulants, Pentoxifylline (100 mg), Complamin (150 mg), Inosine (200 mg), Nootropil (Piracetam, 1200 mg), or Gliatilin (1000 mg). Initially, the administration was intravenous, with a drop counter in the range of 10-15, while later, it was in the form of pills. The courses of the therapy were repeated twice a year.

The results of the treatment of the test group patients are illustrated with Figure 1, Figure 2, Figure 3, Figure 4.

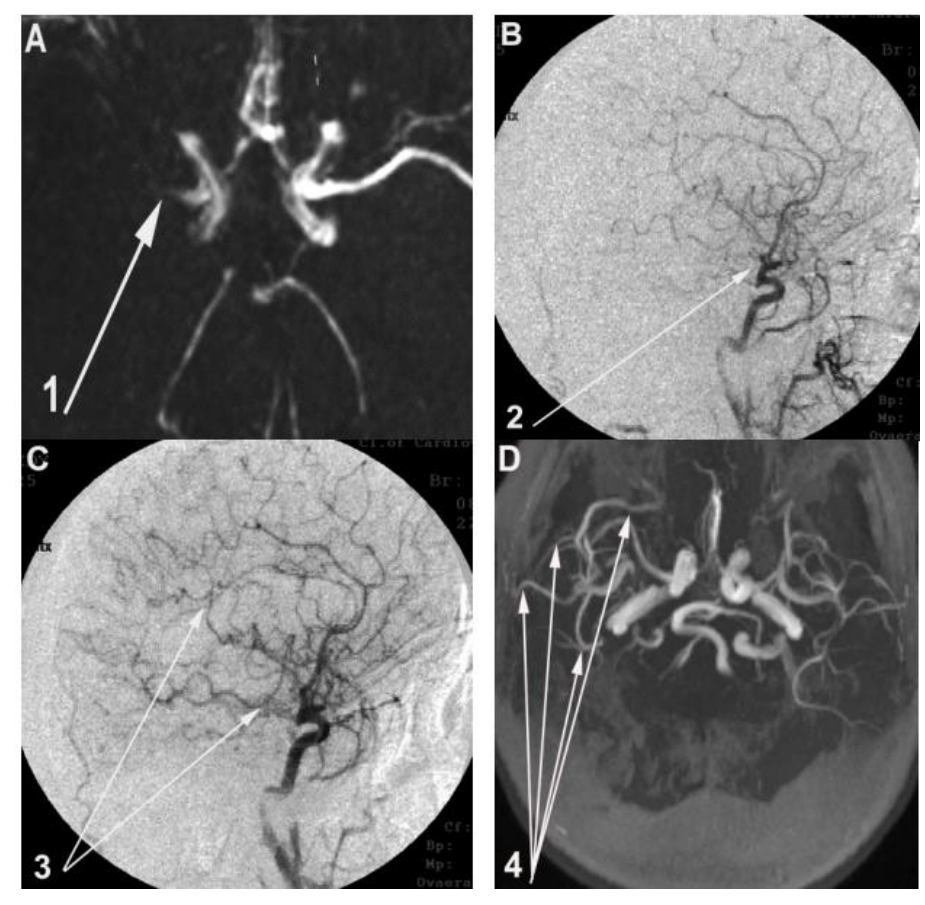

Figure 1 Patient J., male, 41 years old, (BI 40). Atherosclerosis of cerebral vessels, macro focal ischemic stroke of the right hemisphere prior to and after the transcatheter intracerebral laser PBMT. A MRA of the brain, in the initial days after stroke development: 1. Occlusion of the right middle cerebral artery; B After 6 months. Right internal carotid artery angiogram, arterial phase, prior to the transcatheter intracerebral laser PBMT: 2. Occlusion of the right middle cerebral artery; C Right internal carotid artery angiogram, arterial phase, after the transcatheter intracerebral laser PBMT: 3. Pronounced collateral and capillary revascularization of the right hemisphere, with good peripheral blood flow; D MRA of the brain, 10 years after the transcatheter intracerebral laser PBMT: 4. Preservation and progression of the pronounced collateral revascularization of the right hemisphere, with good peripheral blood flow (BI 100). 

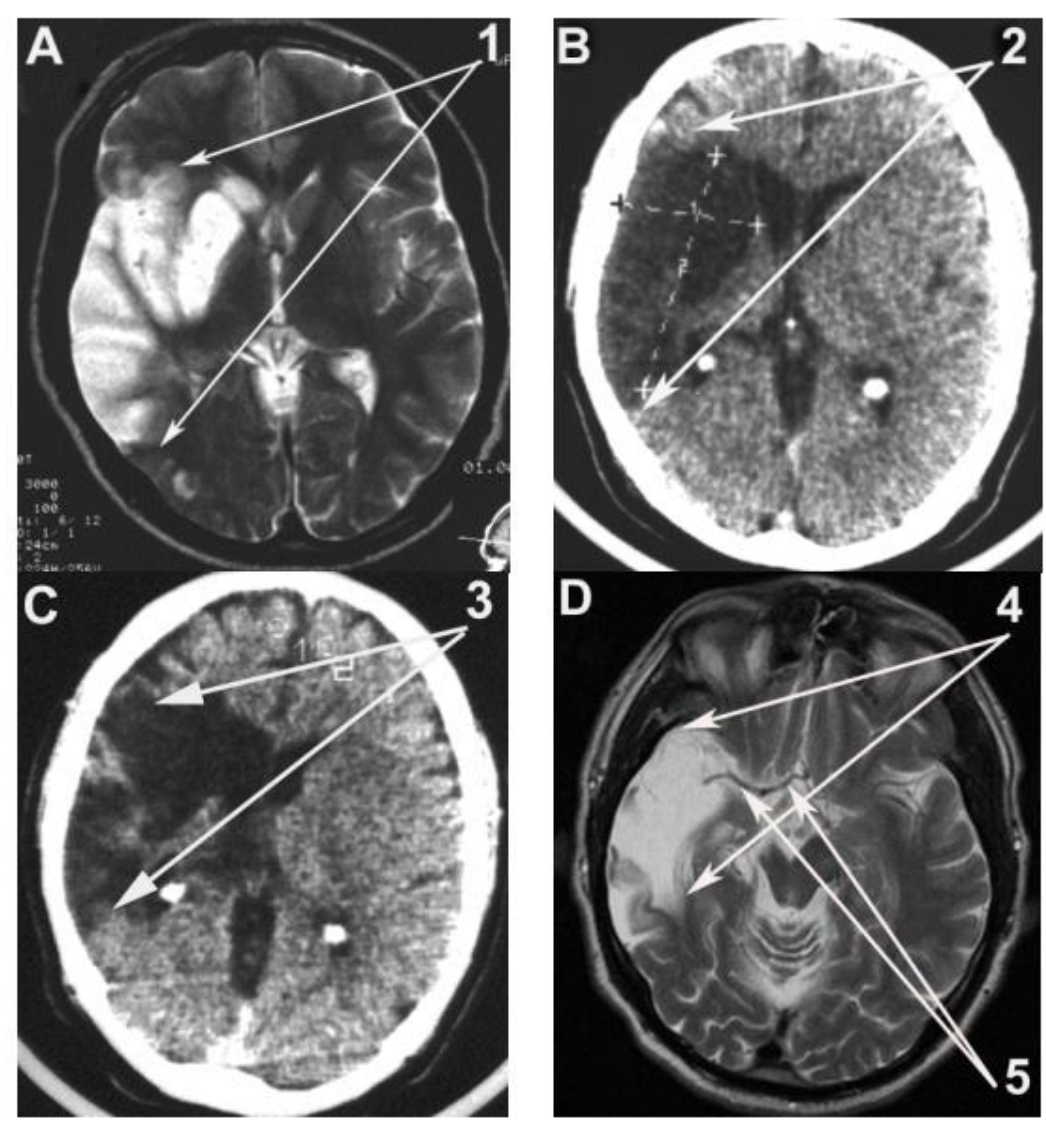

Figure 2 Same patient J., male, 41 years old, (BI 40). Atherosclerosis of cerebral vessels, macro focal ischemic stroke of the right hemisphere, prior to and after the transcatheter intracerebral PBMT. A MRI of the brain, the initial days after stroke development: 1. Formation of an extensive postischemia cyst in the right hemisphere; B After 6 months. $\mathrm{CT}$ of the brain prior to the transcatheter intracerebral laser PBMT: 2. Extensive postischemia cyst in the right hemisphere; C CT of the brain, 12 months after the transcatheter intracerebral laser PBMT: 3. Significant reduction in the size of the postischemic cyst along with cerebral tissue regeneration (BI 95); D MRI of the brain, 10 years after the transcatheter intracerebral laser PBMT: 4. Further diminishing of the postischemic cyst, with cerebral tissue regeneration; 5. Functioning branches of the right middle cerebral artery (BI 100). 

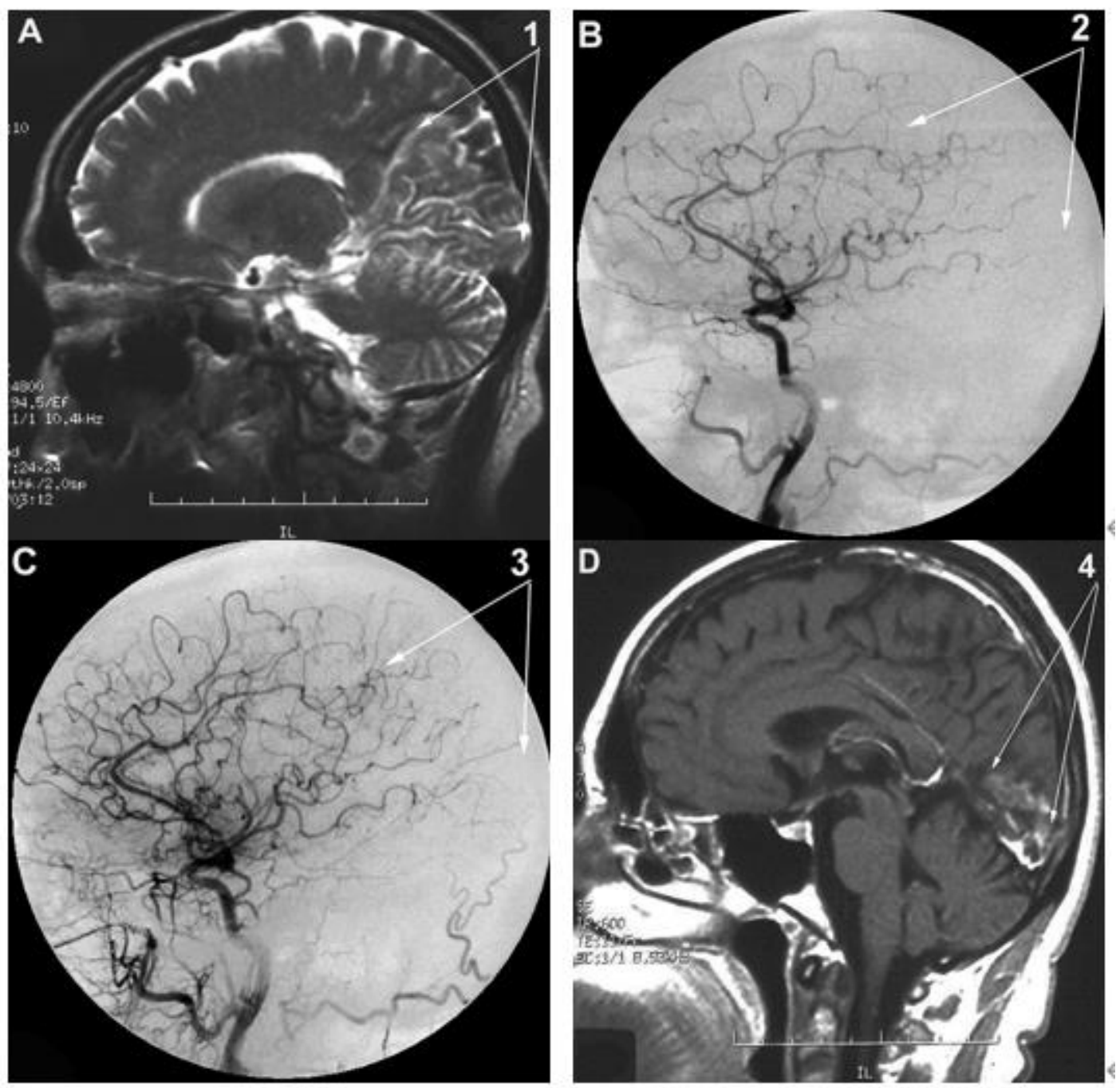

Figure 3 Patient A., female, 58 years old, (BI 50). Atherosclerosis of cerebral vessels, macro focal ischemic stroke of the right hemisphere in the parietal-occipital region, prior to and after the transcatheter intracerebral laser PBMT. A MRI of the brain, prior to the transcatheter intracerebral laser PBMT: 1. A huge post-ischemic cyst in the right parietal-occipital region of the right hemisphere; B Right internal carotid artery angiogram, arterial phase, prior to the transcatheter intracerebral laser PBMT: 2. Multiple occlusions of the distal branches of the right frontal cerebral artery; C Right internal carotid artery angiogram, arterial phase, after the transcatheter intracerebral laser PBMT: 3. Stimulation of angiogenesis, marked collateral arterial and capillary revascularization in the right hemisphere; D MRI of the brain, 10 months after the transcatheter intracerebral laser PBMT: 4. Significant reduction in the size of the postischemic cyst, with signs of cerebral tissue structure recovery (BI 95). 

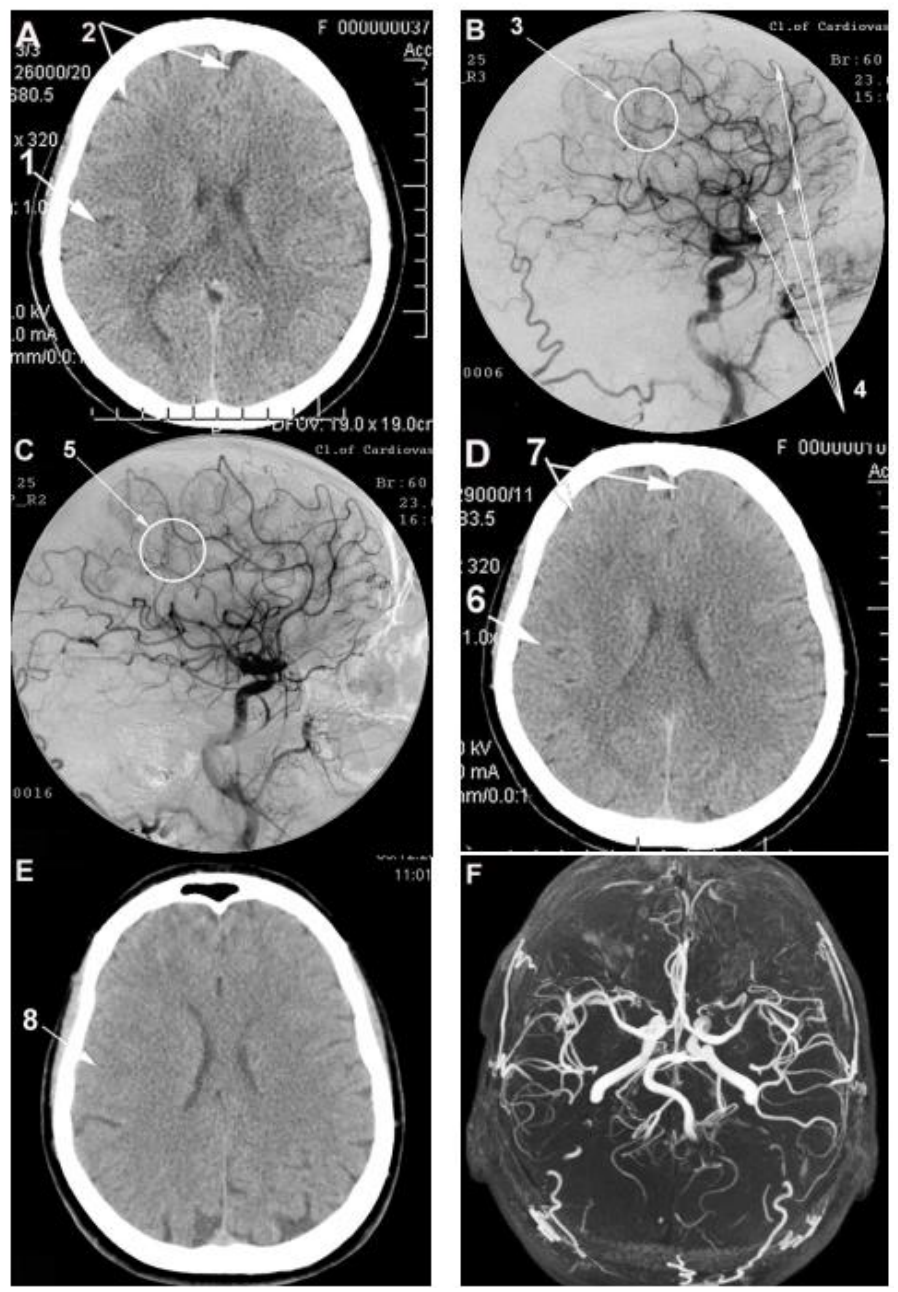

Figure 4 Patient $\mathrm{H}$., female, 60 years old (BI 70). Atherosclerosis of cerebral vessels, chronic cerebrovascular insufficiency, ischemic medium focal stroke in the basin of the middle cerebral artery to the right, prior to and after the transcatheter intracerebral PBMT. A CT of the brain, prior to the transcatheter intracerebral laser PBMT: 1. Medium focal post-ischemic cyst in the right middle cerebral artery region. 2. Moderate expansion of the subarachnoid space; $\mathbf{B}$ Right internal carotid artery angiogram, arterial phase, prior to the transcatheter intracerebral laser PBMT: 3. Occlusion of the distal branches of the right middle cerebral artery; 4 . Multi-focal stenosis of the intracranial branches; C Right internal carotid artery angiogram, arterial phase, after the transcatheter intracerebral laser PBMT: 5. Stimulation of angiogenesis and collateral arterial and capillary revascularization in the right hemisphere; D CT of the brain, 12 months after the transcatheter intracerebral laser PBMT: 6. Reduction in the size of the post-ischemic cyst, with signs of cerebral tissue structure recovery; 7. Subarachnoid space restoration (BI 90); E CT of the brain, six years after the transcatheter intracerebral laser PBMT: 8. The structure of the right hemisphere cerebral tissue was restored, with no signs of the residual effects of the post-ischemic cyst (BI 100); F MRA of the brain. six years after the transcatheter intracerebral laser PBMT: the patency and lumen of the distal branches of the right internal carotid artery were completely preserved, with further progression of collateral revascularization (BI 100). 


\subsubsection{Control Group}

Conservative treatment received by the control group patients included the drugs, regimens, and doses similar to those used for the test group patients. The patients received Aspirin, Heparin, nondirect anticoagulants, Pentoxifylline (100 mg), Complamin (150 mg), Inosine (200 mg), Nootropil (Piracetam, $1200 \mathrm{mg}$ ), or Gliatilin (1000 mg). Initially administered intravenously with a drop counter in the range of 10-15, and later administered as pills. Again, the courses of the therapy were repeated twice a year.

The results of the treatment of the control group patients are illustrated with Figure 5.
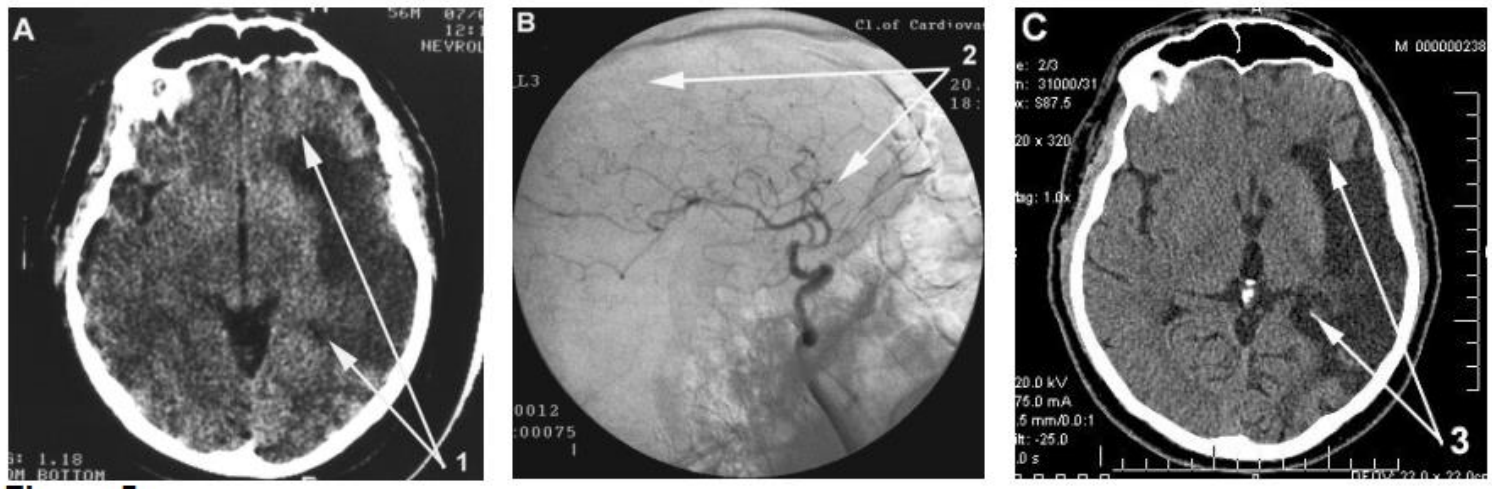

Figure 5 Patient G., male, 56 years old, (BI 40). Atherosclerosis of cerebral vessels, macro focal ischemic stroke of the left hemisphere, prior to and after the conservative treatment. A CT of the brain prior to receiving the conservative treatment: 1. Extensive post-ischemia cyst in the left hemisphere; B Left internal carotid artery angiogram, arterial phase, prior to receiving the conservative treatment: $\mathbf{2}$. Occlusion of the left frontal cerebral artery; C CT of the brain at 2 years after the commencement of the conservative treatment: 3. Extensive post-ischemia cyst in the left hemisphere retained the same size (BI 40).

\subsection{Evaluation of the Clinical Results}

The evaluation of the clinical results achieved after the treatment was conducted in the period between 1 and 10 years. The following were the evaluation criteria:

- good clinical results - almost complete restoration of mental and motor functions and dailylife activities (BI 95-100 and MMSE 27-30);

- satisfactory clinical results - incomplete restoration of mental and motor functions and dailylife activities (BI 85-90 and MMSE 25-27);

- relatively satisfactory clinical results - partial restoration of mental and motor functions and daily-life activities (BI below 80 and MMSE below 25);

- relatively positive clinical results - absence of negative dynamics with little restoration of mental and motor functions and daily-life activities.

\subsection{Statistical Analysis}

The result data were processed statistically using the Statsoft Statistica 10 software (StatSoft Inc., USA). 


\section{Results}

\subsection{Test Group}

\subsubsection{Immediate Results}

After conducting the transcatheter intracerebral laser PBMT, 508 (99.41\%) patients exhibited positive MUGA data results: marked angiogenesis along with collateral and capillary revascularization (Figure $1 \mathrm{~A}-\mathrm{C}$; Figure $3 \mathrm{~B}, \mathrm{C}$; Figure $4 \mathrm{~B}, \mathrm{C}$ ).

No complications associated with these interventions were observed.

\subsubsection{Early Period (1-6 months) after the Transcatheter Intracerebral Laser PBMT}

- clinically, all 511 (100\%) patients exhibited improvement in the motor and mental functions;

- SG and REG results demonstrated improved blood flow and volumetric pulse blood supply in the brain for all 511 (100\%) cases;

- CT and MRI results of all $511(100 \%)$ cases demonstrated decreasing total involutive brain changes and a tendency for the narrowing of the subarachnoid space.

\subsubsection{Later Period (1-10 years) after the Transcatheter Intracerebral Laser PBMT}

- In the first to second- year after the transcatheter intracerebral laser PBMT, all 511 (100\%) patients exhibited the maximum positive clinical results along with the restoration of the motor and mental functions. Moreover, this effect was maintained during the entire period of observation in all 511 (100\%) cases, with no negative dynamics observed (Table 2);

- SG and REG: -the positive result obtained in the early period was maintained in all $511(100 \%)$ cases during the first to second year, and further continued during the entire period of observation;

$\mathrm{CT}$ and MRI results during the evaluations conducted in 1-2 years after the treatment revealed the following:

- decrease in the general involutive brain changes in 489 (95.69\%) cases (Figure 2 A-C; Figure 3 A, D; Figure 4 A, D);

- narrowing of the Sylvian fissures in 473 (92.56\%) cases;

- signs of decreasing non-occlusal hydrocephalus in 326 (63.80\%) cases;

- decrease in the size of the post-ischemic cysts in 475 (92.95\%) cases (Figure $2 \mathrm{~A}-\mathrm{C}$; Figure $3 \mathrm{~A}$, D; Figure 4 A, D), among which- 54 (11.37\%) cases presented a decrease of $10 \%-15 \%,-163$ (34.32\%) cases presented a decrease of $15 \%-25 \%$, and 258 (54.32\%) cases presented a decrease of $>25 \%$. In the examination period beyond 2 years, positive dynamics indicating further restoration of the cerebral tissue were observed in the entire observation period (Figure $2 \mathrm{D}$, Figure $4 \mathrm{E}$ ).

MUGA, MSCTA, and MRA: In the period from two to 10 years after the treatment, among the $193(37.78 \%)$ patients who underwent re-examination, signs of angiogenesis and collateral and capillary revascularization persisted in 191 (98.96\%) cases (Figure 1 D, Figure 4 F). 
Table 2 Clinical results 1-10 years after the treatment for the test group and control group patients.

\begin{tabular}{|c|c|c|c|}
\hline Treatment clinical result & $\begin{array}{l}\text { Test group } \\
\mathrm{n}=511\end{array}$ & $\begin{array}{l}\text { Control group } \\
n=325\end{array}$ & $\mathrm{p}$ (chi-square) \\
\hline \multicolumn{4}{|c|}{ Patients who have had small focal strokes } \\
\hline Good clinical result & 259 & 51 & $<0.005$ \\
\hline Satisfactory clinical result & 33 & 60 & $<0.005$ \\
\hline Relatively satisfactory clinical result & 5 & 102 & $<0.005$ \\
\hline Relatively positive clinical result & 0 & 29 & $<0.005$ \\
\hline Total & 297 & 242 & \\
\hline \multicolumn{4}{|c|}{ Patients who have had medium focal strokes } \\
\hline Good clinical result & 94 & 0 & $<0.005$ \\
\hline Satisfactory clinical result & 39 & 8 & $<0.005$ \\
\hline Relatively satisfactory clinical result & 23 & 10 & $<0.005$ \\
\hline Relatively positive clinical result & 0 & 24 & $<0.005$ \\
\hline Total & 156 & 42 & \\
\hline \multicolumn{4}{|c|}{ Patients who have had macro focal strokes } \\
\hline Good clinical result & 12 & 0 & $<0.005$ \\
\hline Satisfactory clinical result & 22 & 0 & $<0.005$ \\
\hline Relatively satisfactory clinical result & 24 & 7 & $<0.005$ \\
\hline Relatively positive clinical result & 0 & 34 & $<0.005$ \\
\hline Total & 58 & 41 & \\
\hline
\end{tabular}

Since the criteria and information regarding the processing of statistical data are wellrecognized in medical research and practice, the differences between the two groups of patients were determined based on the analysis of the relevant $2 \times 2$ contingency tables using Pearson's chi-squared test. The corresponding $p$-values are listed in the last column of the table. $p=0.05$ was the significance threshold.

\subsubsection{Long-Term Clinical Results}

Long-term clinical results after the treatment obtained for the test group are presented in Table 2.

\subsection{Control Group}

\subsubsection{Immediate Results}

After the first course of the conservative treatment, none of the $325(100 \%)$ patients exhibited negative dynamics, 74 (22.77\%) patients exhibited stabilization of their condition, and 251 (77.23\%) 
patients exhibited a few positive dynamics manifesting as a moderate decrease in motor and mental disorders.

\subsubsection{Early Period (1-6 months) after the Conservative Treatment}

- Clinically, improvement in the motor and mental functions and increase in the $\mathrm{BI}$ were observed in 229 (94.63\%) patients after small focal strokes, 11 (26.19\%) patients after medium focal strokes, and 7 (17.07\%) patients after macro-focal strokes;

- SG and REG: Improvement in the blood flow and volumetric pulse blood supply in the brain were observed in 194 (80.17\%) patients after small focal strokes, 14 (33.33\%) patients after medium focal strokes, and $8(19.51 \%)$ patients after macro-focal strokes, although these improvements were not stable;

- CT and MRI: There was no evidence of any significant decrease in the involutive changes in the brain.

\subsubsection{Long-Term Period (1-10 years) after the Conservative Treatment}

- In the duration of 1-2 years after the treatment, improvement in the motor and mental functions was observed in 122 (50.41\%) patients after small focal strokes, 12 (28.57\%) patients after medium focal strokes, and $6(14.63 \%)$ patients after macro-focal strokes. The remaining patients exhibited stabilization of their condition. In a further distant period, all patients exhibited a decrease in the BI and MMSE (Table 2);

- SG and REG: In the duration of 1-2 years after treatment, the obtained results decreased in 108 (55.67\%) patients among the 194 patients with small focal strokes, six (42.86\%) patients among the 14 patients with medium focal strokes, and three $(37.50 \%)$ patients among the eight patients with macro-focal strokes. The remaining patients exhibited instability of their condition. Afterward, all the patients demonstrated a downward trend.

$\mathrm{CT}$ and $\mathrm{MRI}$ scans conducted in the period of 1-2 years after the treatment revealed the following:

- no significant decrease in the involutive changes occurring in the brain tissue;

- no decrease in the volume of post-ischemic cysts after experiencing the stroke (Figure 5A and $5 \mathrm{C})$;

- in the observation period beyond 2 years, the involutive changes in the brain, the expansion of the Sylvian fissures, the signs of non-occlusal hydrocephalus, and the vascular disorders had intensified in 235 (72.31\%) patients.

\subsubsection{Long-Term Clinical Results}

The long-term clinical results obtained after the treatment for the control group are listed in Table 2.

\section{Discussion}

The present research considered the possibility of treating the consequences of ischemic strokes with the complication of distal intracerebral atherosclerotic lesions. The patients included in the test and control group had similar sizes, conditions, and severity of ischemic strokes. 
In the control group, the SG, REG, CT, and MRI analyses conducted at different time points after the treatment confirmed that the modern therapeutic drugs used in the conservative treatment of stroke are unable to induce marked cerebral revascularization, restoration of the metabolism in neurons, or tissue regeneration [7, 11, 18]. As a consequence, when the patients who had experienced an ischemic stroke of a varying degree of severity were analyzed, good and satisfactory levels of clinical results were obtained in a limited number of patients with small and medium focal ischemic strokes and these results were not stable (Figure 5A and 5C; Table 2).

In the test group, transcatheter intracerebral PBMT was conducted to treat the patients after an ischemic stroke. The intervention was performed using catheters and flexible fiber-optic instruments with a diameter of 25-100 micrometers that were capable of introducing laser energy with a low output power into distal vascular sections. The insignificant thickness of these fiber-optic instruments allowed operating on the intracerebral vessels with a small diameter, rendering this method notably different from the other interventions.

In transcatheter intracerebral PBMT, the energy of the helium-neon laser extends to $20-40 \mathrm{~mm}$ $[5,41,48]$. Therefore, the impact of the laser energy exposure reaches the vascular wall, the blood, and considerably large areas of the cerebral tissue. This impact of the laser energy stimulates angiogenesis and causes rapid opening of arterial collateral and capillary beds, which leads to tissue revascularization in both ischemic and surrounding regions (Figure 1 A-C; Figure 3 B, C; Figure 4 B, C). In addition, the impact of the helium-neon laser on the cerebral tissues restores the metabolic processes and stimulates neurogenesis and tissue regeneration. Since atherosclerosis is a systemic disease that affects, to varying degrees, the entire cerebral arterial bed, the transcatheter intracerebral PBMT was performed from two directions [4, 5, 44, 48]. Laser irradiation of the region where the stroke had developed and the contralateral side region improved blood circulation and tissue metabolism in the two hemispheres of the brain which facilitated rapid physiology-based rehabilitation of the patients. Repeated SG, REG and angiographic examinations in the distant period after the transcatheter intracerebral PBMT revealed that pronounced collateral and capillary revascularization persisted for ten years or beyond (Figure $1 \mathrm{D}$ and Figure $4 \mathrm{~F}$ ).

The CT and MRI scans revealed that after the transcatheter intracerebral laser PBMT, the size of the post-ischemic cyst had decreased, which indicated the recovery of tissue metabolism and development of the regenerative processes in the cerebral tissue (Figure 2 B-D; Figure 3 A, D; Figure $4 \mathrm{~A}, \mathrm{D}, \mathrm{E})$.

In the test group, the processes of angiogenesis, neurogenesis, and regeneration of the cerebral tissue were stimulated in the patients of all age groups, irrespective of the severity or timing of the ischemic stroke and the size of the post-ischemic cyst.

In the test group, good and satisfactory levels of clinical results along with the restoration of motor, mental, and cognitive functions were observed in an overwhelming number of patients with small focal strokes and medium focal strokes, and also in most of the patients with macro-focal strokes (Table 2).

The data obtained in the present research confirm the findings of several researchers who have previously demonstrated by conducting different types of PBMT that the use of laser with a low output power in the red spectral region exerts a complex and multicomponent impact on the brain tissue $[4,14,17,18]$, and this impact of the laser energy stimulates angiogenesis and neurogenesis, which leads to rapid and complete rehabilitation of the patients $[5,31-37,40,41,48]$. 
In the case of an ischemic stroke, disorders in the motor functions are associated with, in addition to brain damage, the developed disorders of the peripheral nervous and muscular systems, which consequently complicates the rehabilitation of the patients. In this regard, it is noteworthy that after conducting the transcatheter intracerebral PBMT, the cognitive and mental functions were restored faster compared to the motor functions, with the latter usually requiring additional treatment.

\section{Conclusions}

Transcatheter intracerebral laser PBMT using a laser with a low output power in the red spectral region represents a pathogenetically and physiologically substantiated, effective method of treating the consequences of ischemic strokes.

The application of this method in patients with ischemic strokes of varying degrees of severity resulting from distal forms of intracerebral atherosclerotic lesions enables the restoration of their daily-life activities and cognitive and mental functions, thereby enabling the patients to be completely active in life once again.

In comparison to PBMT, the conservative methods for treating ischemic strokes did not produce the necessary effective results.

\section{Author Contributions}

The author did all the research work of this study.

\section{Funding}

The author received no financial support for the research, authorship, and/or publication of this article.

\section{Competing Interests}

The author has declared that no competing interests exist.

\section{References}

1. Ahmad FB, Anderson RN. The leading causes of death in the US for 2020. JAMA. 2021; 325: 1829-1830.

2. Abou-Chebl A. Management of acute ischemic stroke. Curr Cardiol Rep. 2013; 15: 348-354.

3. Gillum RF, Kwagyan J, Obisesan TO. Ethnic and geographic variation in stroke mortality trends. Stroke. 2011; 42: 3294-3296.

4. Maksimovich IV. Transcatheter treatment of atherosclerotic lesions of the brain complicated by vascular dementia development. World J Neurosci. 2012; 2: 24664.

5. Maksimovich IV. Transcatheter intracerebral photobiomodulation in ischemic brain disorders: Clinical studies (Part 2). In: Photobiomodulation in the Brain. London: Academic Press is an imprint of Elsevier; 2019. pp.529-544.

6. Pasi M, Cordonnier C. Clinical relevance of cerebral small vessel diseases. Stroke. 2020; 51: 4753. 
7. Brown WR, Thore CR. Cerebral microvascular pathology in ageing and neurodegeneration. Neuropathol Appl Neurobiol. 2011; 37: 56-74.

8. Caplan LR. The effect of small artery disease on the occurrence and management of large artery disease. JAMA Neurol. 2016; 73: 19-20.

9. Zhulev NM, Pustozertsev VG, Zhulev SN. Cerebrovascular diseases. Moscow: Nevsky Dialect; 2002.

10. Pasi M, Poggesi A, Salvadori E, Pantoni L. Post-stroke dementia and cognitive impairment. Front Neurol Neurosci. 2012; 30: 65-69.

11. Pendlebury ST, Wadling S, Silver LE, Rothwell PM. Transient cognitive impairment in TIA and minor stroke. Stroke. 2011; 42: 3116-3121.

12. Polak JF, Pencina MJ, O'Leary DH, D'Agostino RB. Common carotid artery intima-media thickness progression as a predictor of stroke in multi-ethnic study of atherosclerosis. Stroke. 2011; 42: 3017-3021.

13. Schmidtke K, Hull M. Cerebral small vessel disease: How does it progress? J Neurol Sci. 2005; 229: 13-20.

14. Maksimovich IV. Transcatheter cerebral revascularization in the treatment of atherosclerotic lesions of the brain. Brain Disord Ther. 2016; 5: 209.

15. Jin $\mathrm{K}$, Wang $X$, Xie L, Mao XO, Zhu W, Wang Y, et al. Evidence for stroke-induced neurogenesis in the human brain. Proc Natl Acad Sci U S A. 2006; 103: 13198-13202.

16. Galvan V, Jin K. Neurogenesis in the aging brain. Clin Interv Aging. 2007; 2: 605-610.

17. Naeser MA, Hamblin MR. Potential for transcraniallaser or LED therapy to treatstroke, traumatic brain injury, and neurodegenerative disease. Photomed Laser Surg. 2011; 29: 443446.

18. Maksimovich IV. Possibilities of transcatheter treatment of patients after extensive ischemic stroke. World J Neurosci. 2013; 3: 171-185.

19. Altinbas A, Algra A, Brown MM, Featherstone RL, Kappelle LJ, de Borst GJ, et al. Effects of carotid endarterectomy or stenting on hemodynamic complications in the International Carotid Stenting Study: A randomized comparison. Int J Stroke. 2014; 9: 284-290.

20. Takaiwa A, Kuwayama N, Akioka N, Kurosaki K, Hayashi N, Endo S, et al. Effect of carotid endarterectomy on cognitive function in patients with asymptomatic carotid artery stenosis. Acta Neurochir. 2013; 155: 627-633.

21. Featherstone RL, Dobson J, Ederle J, Doig D, Bonati LH, Morris S, et al. Carotid artery stenting compared with endarterectomy in patients with symptomatic carotid stenosis (International Carotid Stenting Study): A randomised controlled trial with cost-effectiveness analysis. Health Technol Assess. 2016; 20: 1-94.

22. Bonati LH, Lyrer P, Ederle J, Featherstone R, Brown MM. Percutaneous transluminal balloon angioplasty and stenting for carotid artery stenosis. Cochrane Database Syst Rev. 2012: 12; CD000515.

23. Lamanna A, Maingard J, Barras CD, Kok HK, Handelman G, Chandra RV, et al. Carotid artery stenting: Current state of evidence and future directions. Acta Neurol Scand. 2019; 139: 318333.

24. Kim NY, Choi JW, Whang K, Cho SM, Koo YM, Kim JY. Neurologic complications in patients with carotid artery stenting. J Cerebrovasc Endovasc Neurosurg. 2019; 21: 86-93. 
25. Haupert G, Ammi M, Hersant J, Daligault M, Tesson P, Papon X, et al. Treatment of carotid restenoses after endarterectomy: A retrospective monocentric study. Ann Vasc Surg. 2020; 64: 43-53.

26. Caplan LR, Thomas AJ, Inoa V. Interventional treatment of brain ischemia related to intracranial cerebrovascular occlusive lesions. Curr Opin Neurol. 2014; 27: 1-7.

27. Gramegna LL, Cardozo A, Folleco E, Tomasello A. Flow-diverter reconstruction of an intracranial internal carotid artery dissection during thrombectomy for acute ischaemic stroke. BMJ Case Rep. 2020; 13: e231612.

28. Fjetland L, Roy S, Kurz KD, Larsen JP, Kurz MW. Endovascular acute stroke treatment performed by vascular interventional radiologists: Is it safe and efficacious? Cardiovasc Intervent Radiol. 2012; 35: 1029-1035.

29. Lee JS, Hong JM, Lee SJ, Joo IS, Lim YC, Kim SY. The combined use of mechanical thrombectomy devices is feasible for treating acute carotid terminus occlusion. Acta Neurochir. 2013; 155: 635641.

30. Ciccone A, Valvassori L, Nichelatti M, Sgoifo A, Ponzio M, Sterzi R, et al. Endovascular treatment for acute ischemic stroke. N Engl J Med. 2013; 368: 904-913.

31. Hashmi JT, Huang YY, Osmani BZ, Sharma SK, Naeser MA, Hamblin MR. Role of low-level laser therapy in neurorehabilitation. PM R. 2010; 2: S292-S305.

32. Hamblin MR. Photobiomodulation for traumatic brain injury and stroke. J Neurosci Res. 2018; 96, 4: 731-743.

33. Saltmarche AE, Naeser MA, Ho KF, Hamblin MR, Lim L. Significant improvement in cognition in mild to moderately severe dementia cases treated with transcranial plus intranasal photobiomodulation: Case series report. Photomed Laser Surg. 2017; 35: 432-441.

34. Hamblin MR. Photobiomodulation, Photomedicine, and laser surgery: A new leap forward into the light for the 21st century. Photomed Laser Surg. 2018; 36: 395-396.

35. Hamblin MR. Mechanisms and mitochondrial redox signaling in photobiomodulation. Photochem Photobiol. 2018; 94: 199-212.

36. Huang YY, Hamblin MR. Photobiomodulation on cultured cortical neurons. In: Photobiomodulation in the Brain. London: Academic Press is an imprint of Elsevier; 2019. pp.3546.

37. Hamblin MR. Mechanisms of photobiomodulation in the brain. In: Photobiomodulation in the Brain. London: Academic Press is an imprint of Elsevier; 2019. pp.97-110.

38. Lapchak PA. The challenge of effectively translating transcranial near-infrared laser therapy to treat acute ischemic stroke. In: Photobiomodulation in the Brain. London: Academic Press is an imprint of Elsevier; 2019. pp.289-298.

39. Taboada LD, Hamblin MR. Transcranial photobiomodulation for stroke in animal models. In: Photobiomodulation in the Brain. London: Academic Press is an imprint of Elsevier; 2019. pp.111-124.

40. Salehpour F, Gholipour-Khalili S, Farajdokht F, Kamari F, Walski T, Hamblin MR, et al. Therapeutic potential of intranasal photobiomodulation therapy for neurological and neuropsychiatric disorders: A narrative review. Rev Neurosci. 2020; 31: 269-286.

41. Maksimovich IV. Method of transluminal laser revascularization of cerebral blood vessels having atherosclerotic lesions. Washington: United States Patent and Trademark Office; 2008; US7490612. 
42. Maksimovich IV. Transljuminal laser angioplasty in treatment of ischemic lesions of a brain. Moscow: Russian University of Friendship of the People; 2004.

43. Maksimovich IV. Method for carrying out transluminal laser-induced brain revascularization in atherosclerotic injury cases. Camden: RF Products, Inc.; 2006; 2297861.

44. Maksimovich IV. Laser technologies as a new direction in transcatheter interventions. Photomed Laser Surg. 2019; 37: 455-456.

45. Mahoney FI, Barthel DM. Functional evaluation: The barthel index. Md State Med J. 1965; 14: 61-65.

46. Morris JC. The Clinical Dementia Rating (CDR): Current version and scoring rules. Neurology. 1993; 11: 2412-2414.

47. Folstein MF, Folstein SE, McHugh PR. "Mini-mental state". A practical method for grading the cognitive state of patients for the clinician. J Psychiatr Res. 1975; 12: 189-198.

48. Maksimovich IV. Intracerebral transcatheter laser PBMT in the treatment of Binswanger's disease and vascular parkinsonism: Research and clinical experience. Photomed and Laser Surg. 2019; 37: 606-614.

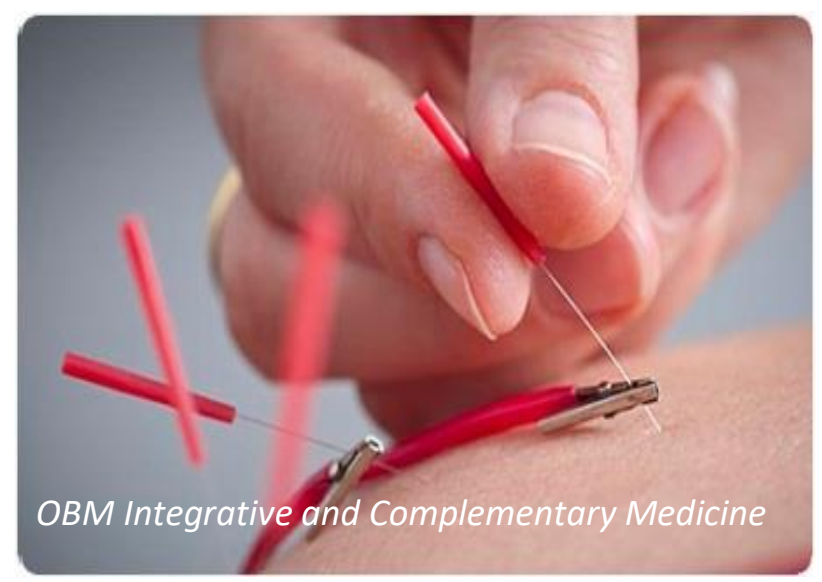

Enjoy OBM Integrative and Complementary Medicine by:

1. Submitting a manuscript

2. Joining in volunteer reviewer bank

3. Joining Editorial Board

4. Guest editing a special issue

For more details, please visit: http://www.lidsen.com/journals/icm 